\title{
A Study of the Relationship between the Tensile Strength and Dynamics of As-spun and Drawn Poly(glycolic acid) Fibers
}

\author{
By Sokei SEKINE, ${ }^{1,3, *}$ Hideyuki AKIEDA, ${ }^{2}$ Isao ANDO ${ }^{4}$ and Tetsuo ASAKURA ${ }^{3}$
}

\begin{abstract}
Higher-order structure of as-spun and highly-drawn poly(glycolic acid)(PGA) fibers has been characterized by solid-echo ${ }^{1} \mathrm{H}$ NMR and WAXD methods. In the observed WAXD patterns, as-spun PGA fiber shows a very broad amorphous peak and highly-drawn PGA fibers show very sharp diffraction peaks at $2 \theta=21.9^{\circ}$ from which the crystallinities of highly-drawn fibers with the drawing ratio $\left(\lambda_{\text {d.r. }}\right)$ of $2.5,2.8$ and 3.2 are determined to be $0.50,0.51$ and 0.52 , respectively. These show that the crystallinities of the PGA fibers with different drawing ratios are very close to each other. On the other hand, the observed solid-echo ${ }^{1} \mathrm{H}$ NMR free induction decay(FID) signals of as-spun and drawn PGA fibers show a multi-component decay consisting of the short $\mathrm{T}_{2}$ component and the long $\mathrm{T}_{2}$ component. From these results, the short $\mathrm{T}_{2}$ component was assigned to the crystalline region and the immobile amorphous region, and the long $\mathrm{T}_{2}$ component was assigned to the mobile amorphous region. It was found that the fractions of the short $\mathrm{T}_{2}$ component and the long $\mathrm{T}_{2}$ component of highly-drawn fibers largely depend on the drawing ratio $\lambda_{\text {d.r. }}$ and temperature, and that the fractions of the short $\mathrm{T}_{2}$ component for drawn PGA fibers with $\lambda_{\text {d.r. }}$ of 2.5 at $120^{\circ} \mathrm{C}$ and $160^{\circ} \mathrm{C}$ are 0.85 and 0.56 , respectively. Further, it was found that the fractions of the short $\mathrm{T}_{2}$ component for the drawn fibers above $160^{\circ} \mathrm{C}$ are very close to the crystallinities determined by the WAXD patterns, but the fractions of the short $\mathrm{T}_{2}$ component at $120^{\circ} \mathrm{C}$ are different from each other. The structural characterization was made to understand mechanical property of the drawn PGA fibers. Then, it was found that the fraction of the corresponding amorphous region is closely related to the ratio of the tensile strength after hydrolytic acceleration test to that before hydrolytic acceleration for PGA fibers.
\end{abstract}

KEY WORDS: Poly(glycolic acid) / Biodegradable Polymer / Solid-state NMR / Solid-echo / Relaxation Time /

Poly(glycolic acid)(PGA) $)^{1-4}$ is one of semi-crystalline biodegradable polyesters such as poly(dioxanone), ${ }^{5-8}$ poly(lactide $)^{9,10}$ and poly( $\varepsilon$-caprolactone). ${ }^{11}$ The fibers of these biodegradable polymers have been successfully used as suture materials. The aspects of biocompatibility of PGA and its copolymers are well documented elsewhere. ${ }^{12,13}$ One of the main disadvantages of PGA is narrow window of processing conditions by degradable properties and crystallization rate, which makes it very difficult to produce thick specimens with adequate mechanical properties. Thus, PGA suture is used as a multi filament made of yarn which bound some of thin fibers.

The hydrolytic degradation of PGA has been studied by examining changes of tensile strength and the level of crystallinity of suture materials. ${ }^{14}$ The sigmoidal shape of the stress-strain curves gradually disappears with increasing duration of in vitro degradation. Although the concept of microfibrillar structure of PGA fibers has been proposed for considering the degradation mechanism of PGA in vitro, it is desirable to understand the structure-properties relationship of PGA in details from atomic-level structural studies such as Xray diffraction or solid-state NMR. Chatani et al. ${ }^{15}$ determined the crystal structure of PGA by X-ray diffraction to have the dimensions of the orthorhombic unit cell with $a=5.22 \AA$, $b=6.19 \AA$ and $c$ (the fiber axis) $=7.02 \AA$. Two PGA chains in planar zig-zag conformation passing through the unit cell permit tight molecular-packing and the close approach of ester groups. de Oca et al. ${ }^{16}$ studied dynamics of isotropic and highly-drawn PGA fibers by second-moment analysis of ${ }^{1} \mathrm{H}$ NMR signal and ${ }^{1} \mathrm{H} \mathrm{T}_{1 \rho}$ analysis at $100^{\circ} \mathrm{C}$ by means of solid-state ${ }^{1} \mathrm{H}$ NMR. They have shown that isotropic PGA sample has two kinds of components such as the rigid crystalline region and mobile amorphous region, but highlydrawn PGA sample has three components consisting of rigid crystalline region with long relaxation time, semi-rigid noncrystalline region with intermediate relaxation time and highly mobile non-crystalline region with the shortest relaxation time. Thus, it can be said that solid-state NMR has provided fruitful information about both structure and dynamics of polymers. ${ }^{17,18}$

From such a background, the present work aims to characterize structure and dynamics of as-spun and highlydrawn PGA fibers at a wide range of temperatures by observation of wide-angle X-ray diffraction(WAXD) pattern, and solid-echo ${ }^{1} \mathrm{H}$ free induction decay(FID) and spin-spin relaxation time $\left(\mathrm{T}_{2}\right)$ by solid-state ${ }^{1} \mathrm{H} \mathrm{NMR}$, in order to clarify a relationship between the retention strength of PGA fibers in buffered solution and the structure/dynamics.

\footnotetext{
${ }^{1}$ Mitsui Chemical Analysis \& Consulting Service, Inc., 580-32, Nagaura, Sodegaura 299-0265, Japan

${ }^{2}$ Nagoya Works, Engineered Materials Manufacturing Department, Mitsui Chemicals, Inc., 2-1 Tango-dori, Minami-ku, Nagoya 457-0801, Japan

${ }^{3}$ Department of Biotechnology, Tokyo University of Agriculture and Technology, Koganei 184-8588, Japan

${ }^{4}$ Tokyo Institute of Technology, Meguro-ku, Tokyo 152-8552, Japan

*To whom correspondence should be addressed (Tel: +81-438-64-2406, Fax: +81-438-64-2407, E-mail: Sokei.Sekine@mitsui-chem.co.jp).
} 


\section{MATERIALS AND METHODS}

\section{Materials}

PGA was synthesized from glycolide used as starting compound by Mitsui Chemicals, Inc. and its molecular weight was determined to be about $2 \times 10^{5}$ using GPC method. PGA fibers were prepared at $230^{\circ} \mathrm{C}$ from the molten state. After cooling them at room temperature, resulting fibers were heated again at a temperature just above the glass transition temperature of PGA $\left(T_{\mathrm{g}}=60{ }^{\circ} \mathrm{C}\right)$, and were stretched by $2.3,2.8$ or 3.2 times to obtain highly-drawn PGA fiber samples. The drawing ratio $\left(\lambda_{\text {d.r. }}\right)$ of PGA fibers was defined to be the ratio of stretched fiber length to original untreated fiber length.

\section{Tensile Strength Measurements}

PGA fibers of a definite weight of $0.0112 \sim 0.0136 \mathrm{~g}$ were soaked into phosphate buffer solution at $50{ }^{\circ} \mathrm{C}$ for $3 \mathrm{~d}$ and then were set on a tensile test machine covered with a filter paper. The tensile strength was measured at room temperature with an extension rate of $100 \mathrm{~mm} / \mathrm{min}$ under the gauge length of the sample was kept at $100 \mathrm{~mm}$. The measurement was repeated 10 times, and then the averaged tensile strength and the averaged stretched length of fiber broken under strain were determined. The tensile strength $\left(\mathrm{T}_{\text {single }}\right)$ of a single fiber was estimated by the following equation.

$$
\mathrm{T}_{\text {single }}=\mathrm{G} / \mathrm{d}
$$

where $G$ is the tensile breaking force and $d$ is the fineness. In analysis of the tensile strength of drawn PGA fibers with hydrolytic acceleration test, the relative tensile strength is used. The relative tensile strength is defined as the ratio of the tensile strength after hydrolytic acceleration test to that before hydrolytic acceleration for 10 kinds of drawn PGA fibers as mentioned below.

\section{Pulse ${ }^{1}$ H NMR Measurements}

Pulse ${ }^{1} \mathrm{H}$ NMR spectra were recorded by a JEOL JNMMU25 NMR spectrometer operating at $25 \mathrm{MHz}$ for ${ }^{1} \mathrm{H}$ resonance frequency. The solid-echo signal was obtained by using $(\pi / 2)_{\mathrm{x}}-\tau-(\pi / 2)_{\mathrm{y}}$ pulse sequence, where $\tau$ is the pulse interval between $(\pi / 2)_{\mathrm{x}}$ pulse and $(\pi / 2)_{\mathrm{y}}$ pulse. Variabletemperature solid-echo ${ }^{1} \mathrm{H}$ NMR measurements were made in the temperature range from 80 to $200{ }^{\circ} \mathrm{C}$ with an interval of $20^{\circ} \mathrm{C}$. Highly-drawn PGA fibers of about $100 \mathrm{mg}$ were coiled around a glass stick with $10 \mathrm{~mm}$ width. Then, this glass stick coiled by the PGA fibers is placed in an NMR magnet. The magnetic field is applied to the direction perpendicular to the glass stick. This means that the directions of proton-proton vectors of PGA in the fibers are isotropically distributed in the static magnetic field. This becomes the same state such that a number of PGA pieces cut into small size are randomly placed into an NMR tube. It has been recognized that the observed solid-echo FID signals of these two kinds of PGA samples are almost same to each other. The observed FID signal (I $(\mathrm{t}))$ of the PGA fibers, in principle, should be analyzed with function
$\mathrm{I}(\mathrm{t})=\mathrm{A} \exp \left\{-(1 / 2) \mathrm{kt}^{2}\right\} \sin (b \mathrm{t}) / b \mathrm{t}$ in which molecular motion of PGA chains is strongly restrained. It is known that this function becomes Gaussian function when $b=0$ and so $\sin (b t) / b t=1$. The observed FID signal was fitted by using this function. It was found that the FID signal can be fitted by Gaussian function. Therefore, $b$ should be 0 , and then all of the observed FID signals were analyzed by Gaussian function. At high temperatures the PGA chains are in the mobile state and then the FID signals are fitted by Lorentzian function. Therefore, all of the observed ${ }^{1} \mathrm{H}$ FID signals were fitted by a sum of Gaussian function and Lorentzian function corresponding to the short $\mathrm{T}_{2}$ component and the long $\mathrm{T}_{2}$ component, respectively, by using a JEOL software.

\section{X-Ray Diffraction Measurements}

Wide-angle X-ray diffraction(WAXD) of PGA fibers were measured by a Rigaku RINT2500 X-ray instrument utilizing nickel-filtered $\mathrm{Cu} \mathrm{K}_{\alpha}$ radiation(wave length $\lambda=1.54 \AA$ ) at room temperature. PGA fiber samples were rolled and mounted on a holder.

\section{RESULTS AND DISCUSSION}

\section{Structure of PGA Fibers Characterized by Wide-angle X-ray Diffraction (WAXD)}

Figure 1 shows the observed WAXD patterns of as-spun PGA fiber and drawn PGA fibers with $\lambda_{\text {d.r. }}=2.5,2.8$ and 3.2. ${ }^{19,20}$ The diffraction pattern of as-spun PGA fiber shows a very broad amorphous peak alone in the absence of a sharp crystalline peak, which comes from the amorphous region. Thus, this means that the crystallinity of as-spun fiber is extremely low. On the other hand, the relatively sharp diffraction peaks of these drawn fibers were observed at $2 \theta=21.9^{\circ}$ and $28.8^{\circ}$. The crystallinities of drawn PGA fibers with $\lambda_{\text {d.r. }}=2.5,2.8$ and 3.2 were determined to be $0.50,0.51$ and 0.52 , respectively, and the degrees of orientation were $0.92,0.93$ and 0.94 , respectively. In spite of a large change in

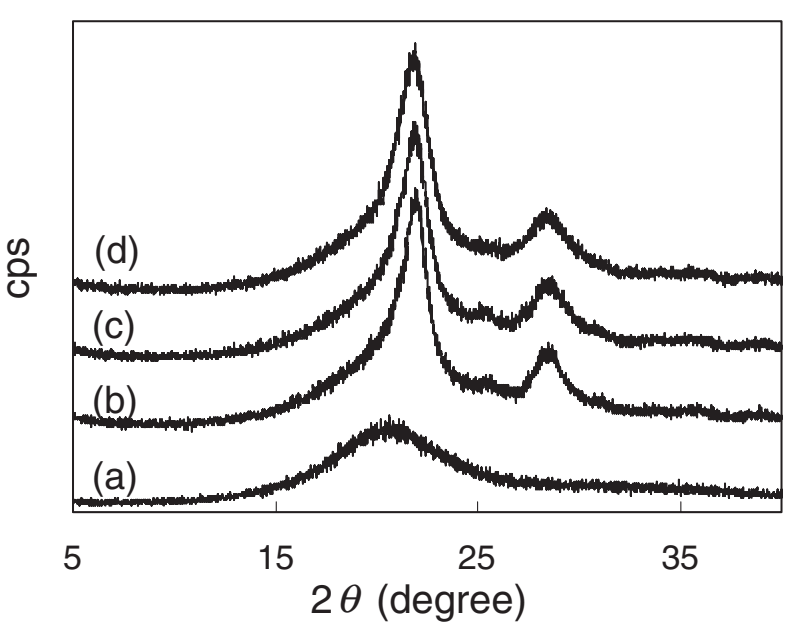

Figure 1. X-Ray diffraction patterns of PGA fibers. As-spun PGA fiber(a) and drawn PGA fibers with the drawing ratios, $\lambda_{\text {d.r. }}=2.5(\mathrm{~b}), 2.8(\mathrm{c})$ and $3.2(d)$. 
the drawing ratio, the degrees of crystallinity and orientation do not change largely. However, it is noted that the width of the diffraction peak increases slightly with an increase in the drawing ratio, indicating that the local structure in the crystalline region of the drawn PGA fibers was slightly disturbed with an increase in the drawing ratio.

\section{Structure and Dynamics of PGA Fibers Characterized by Pulse ${ }^{1}$ H NMR}

In order to clarify effect of the drawing on structure and molecular motion of PGA fiber, solid-echo ${ }^{1} \mathrm{H}$ FID signals of drawn PGA fibers were observed as a function of $\lambda_{\text {d.r. }}$. The observed FID signal of PGA fiber with $\lambda_{\text {d.r. }}=2.5$ at $120^{\circ} \mathrm{C}$ as indicated by black solid line (Figure 2(a)) was reasonably deconvoluted into two curves indicated by gray solid line as shown in Figure 2(b) and 2(c). They are referred to the short $\mathrm{T}_{2}$ component (decay (b)) and the long $\mathrm{T}_{2}$ component (decay (c)), respectively. The curves for the short $T_{2}$ component and the long $\mathrm{T}_{2}$ component follow a Gaussian function and a Lorentzian function, respectively. The experimental results of the short $\mathrm{T}_{2}$ component and the long $\mathrm{T}_{2}$ component of PGA fiber with $\lambda_{\text {d.r. }}=2.5$ as a function of temperature are shown in Table I. The fraction of the short $\mathrm{T}_{2}$ component decreases largely in going from 120 to $160^{\circ} \mathrm{C}$, and becomes a plateau at temperatures from 160 to $200^{\circ} \mathrm{C}$. The observed solid-echo ${ }^{1} \mathrm{H}$ FID signal of drawn PGA fiber with $\lambda_{\text {d.r. }}=2.5$ at $160^{\circ} \mathrm{C}$ is shown in Figure 3. The observed FID signal indicated by (a) is deconvoluted into two curves (b) (short $\mathrm{T}_{2}$ component) and (c) (long $\mathrm{T}_{2}$ component) indicated by the gray solid line. From this

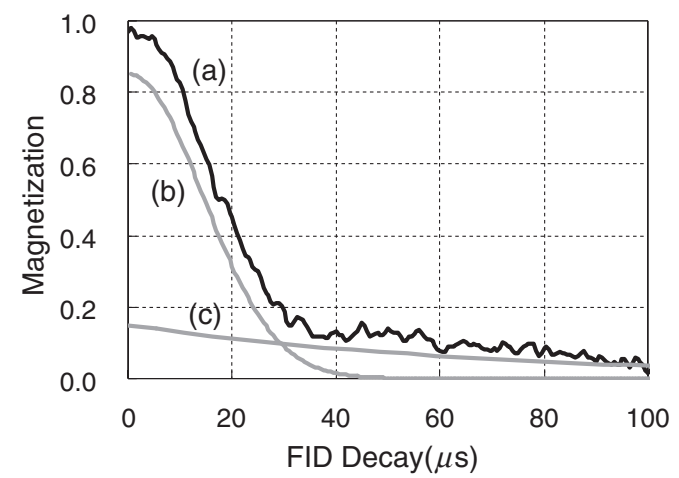

Figure 2. Solid-echo ${ }^{1} \mathrm{H}$ FID signal of drawn PGA fiber with the drawing ratio, $\lambda_{\text {d.r. }}=2.5$ at $120^{\circ} \mathrm{C}$. Observed signal(a), and deconvoluted signals (short $\mathrm{T}_{2}$ decay: (b) and long $\mathrm{T}_{2}$ decay: (c)).

Table I. The fractions and $T_{2}$ values of the short $T_{2}$ component and the long $T_{2}$ component of PGA fibers with drawing ratio $\lambda_{\text {d.r. }}=2.5$ obtained from deconvolution of solid-echo ${ }^{1} \mathrm{H}$ FID signal as a function of temperature

\begin{tabular}{lccccc}
\hline temperature $\left({ }^{\circ} \mathrm{C}\right)$ & 120 & 140 & 160 & 180 & 200 \\
\hline short $\mathrm{T}_{2}$ component & & & & & \\
fraction & 0.85 & 0.60 & 0.56 & 0.52 & 0.52 \\
$\mathrm{~T}_{2}$ value $(\mu \mathrm{s})$ & 19.8 & 18.8 & 18.5 & 18.6 & 18.6 \\
& & & & & \\
long $\mathrm{T}_{2}$ component & & & & & \\
fraction & 0.15 & 0.40 & 0.44 & 0.48 & 0.48 \\
$\mathrm{~T}_{2}$ value $(\mu \mathrm{s})$ & 55.7 & 60.7 & 69.7 & 76.0 & 84.5 \\
\hline
\end{tabular}

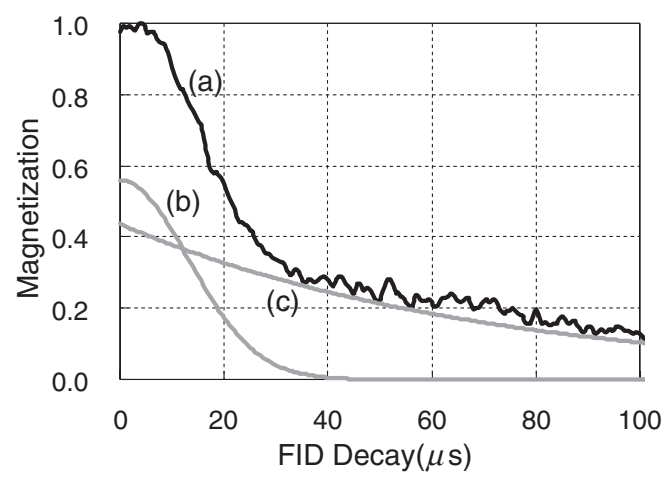

Figure 3. Solid-echo ${ }^{1} \mathrm{H}$ FID signals of drawn PGA fiber with the drawing ratio, $\lambda_{\text {d.r. }}=2.5$ at $160^{\circ} \mathrm{C}$. Observed signal(a), and the deconvoluted signals (short $T_{2}$ decay: (b) long $T_{2}$ decay: (c)).

deconvolution, the fraction of the short $\mathrm{T}_{2}$ component was obtained to be 0.56 . By elevating temperature from 120 to $160{ }^{\circ} \mathrm{C}$, the fraction is decreased by 0.29 from 0.85 to 0.56 . The reduction of the fraction for the short $\mathrm{T}_{2}$ component means an increase in the fraction of the mobile amorphous region as compared with the results of the WAXD pattern. This shows that the fraction of the short $\mathrm{T}_{2}$ component at $160^{\circ} \mathrm{C}$ obtained by pulse ${ }^{1} \mathrm{H}$ NMR and the crystallinity obtained by WAXD to be 0.50 are close to each other. The DSC diagram (Figure 4) shows that there are no melt and no crystallization heat flow in the vicinity of $160^{\circ} \mathrm{C}$, and melt begins at $180^{\circ} \mathrm{C}$. Table II shows the relationship between the fraction of the short $\mathrm{T}_{2}$ component at $160{ }^{\circ} \mathrm{C}$ and the crystallinity obtained by WAXD as a function of $\lambda_{\text {d.r. }}$. There is small difference between them, but both of them are very close to each other. This small

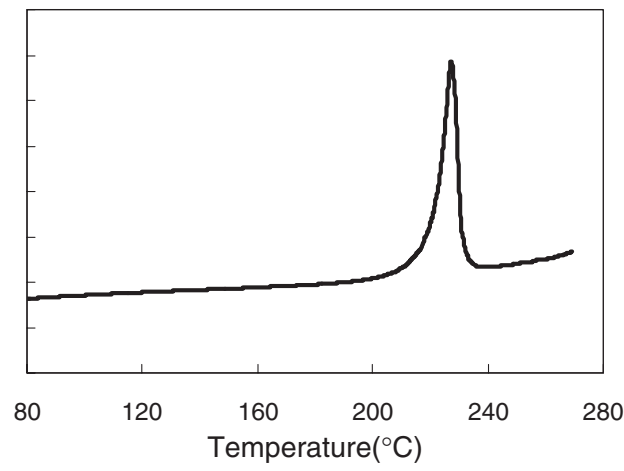

Figure 4. DSC diagram of drawn PGA fiber with drawing ratios, $\lambda_{\text {d.r. }}=2.5$.

Table II. The fraction of the short $T_{2}$ component of PGA fibers obtained by NMR at $160^{\circ} \mathrm{C}$ and the crystallinity obtained by WAXD as a function of drawing ratio

\begin{tabular}{ccc}
\hline $\begin{array}{c}\text { drawing ratio } \\
\lambda_{\text {d.r. }}\end{array}$ & crystallinity(WAXD) & $\begin{array}{c}\text { fraction of short } \mathrm{T}_{2} \text { component } \\
(\mathrm{NMR}) \text { at } 160^{\circ} \mathrm{C}\end{array}$ \\
\hline 2.35 & 0.50 & 0.52 \\
2.50 & 0.49 & 0.52 \\
2.75 & 0.49 & 0.56 \\
2.80 & 0.50 & 0.59 \\
3.05 & 0.51 & 0.59 \\
\hline
\end{tabular}




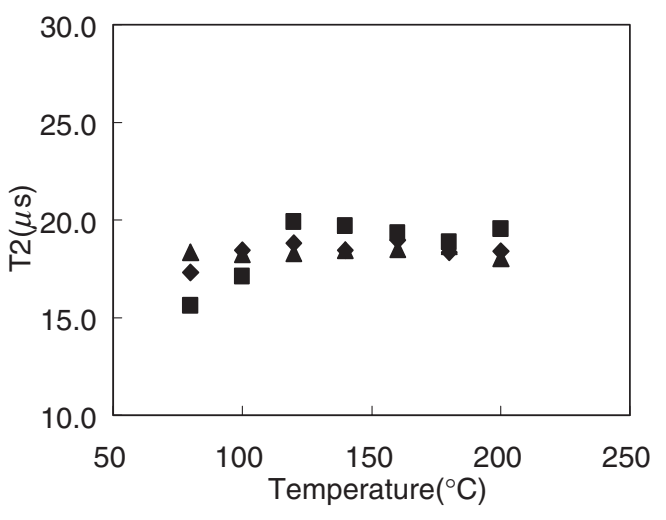

Figure 5. Plots of the value of the short $T_{2}$ for as-spun PGA fiber $(\mathbf{A})$, drawn PGA with the drawing ratio, $\lambda_{\mathrm{dr}}=2.5(\diamond)$ and drawn PGA with the drawing ratio, $\lambda_{\text {d.r. }}=3.05(\square)$ as estimated from deconvolution of the observed ${ }^{1} \mathrm{H}$ FID signals as a function of temperature in the temperature range from 80 to $200^{\circ} \mathrm{C}$

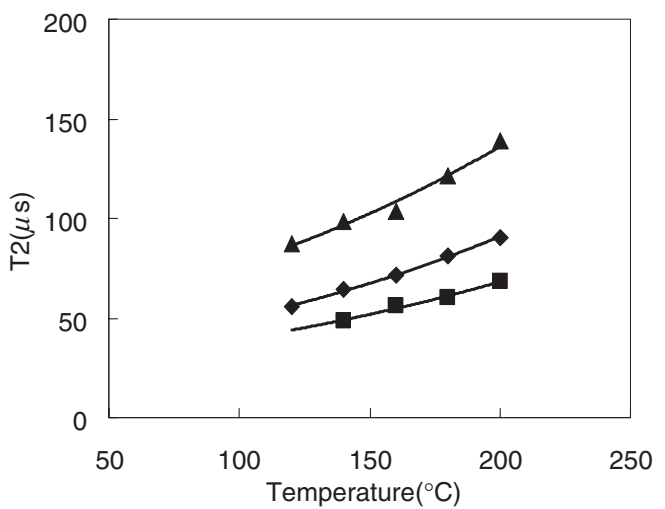

Figure 6. Plots of the value of the long $T_{2}$ for as-spun PGA fiber $(\boldsymbol{\Delta})$, drawn PGA with the drawing ratio, $\lambda_{\text {d.r. }}=2.5(\diamond)$ and drawn PGA with the drawing ratio, $\lambda_{\mathrm{dr}}=3.05(\square)$ as estimated from deconvolution of the observed ${ }^{1} \mathrm{H}$ FID signals as a function of temperature in the temperature range from 80 to $200^{\circ} \mathrm{C}$

difference may come from the fact that NMR detects difference in molecular motion and WAXD detects difference in local structure such as the crystalline and amorphous structures. Then, it can be said that the short $\mathrm{T}_{2}$ component at $160^{\circ} \mathrm{C}$ obtained by NMR almost comes from the crystalline region.

Further, in order to elucidate the local structure of PGA fibers by changing the drawing ratio and temperature, the ${ }^{1} \mathrm{H} \mathrm{T}_{2}$ values and fractions for the short $\mathrm{T}_{2}$ component and the long $\mathrm{T}_{2}$ component of 10 PGA fiber samples with different drawing ratios $\lambda_{\text {d.r. }}$ from 2.30 to 3.05 at 120 and $160^{\circ} \mathrm{C}$ were determined by using the above-mentioned procedure. The former is shown in Figures 5 and 6, and the latter is shown in Tables III and IV. As shown in Figures 5 and 6 , the ${ }^{1} \mathrm{H} \mathrm{T}_{2}$ values for the short $\mathrm{T}_{2}$ component are slowly increased in going from 80 to $120^{\circ} \mathrm{C}$, and become a plateau at temperatures from 120 to $200^{\circ} \mathrm{C}$. Its variation is very small to be in the $17-20 \mu$ s range, and the drawing ratio dependence of ${ }^{1} \mathrm{H} \mathrm{T}_{2}$ is not so large. Obviously, molecular motion for the short $\mathrm{T}_{2}$ component is strongly restrained in the NMR time scale as viewed from the $\mathrm{T}_{2}$ value
Table III. The fractions and $T_{2}$ values of the short $T_{2}$ component and the long $T_{2}$ component obtained from deconvolution of solid-echo ${ }^{1} \mathrm{H}$ FID signal as a function of drawing ratio at $120^{\circ} \mathrm{C}$

\begin{tabular}{|c|c|c|c|c|c|c|c|c|c|c|}
\hline \multirow[b]{2}{*}{ sample No.* } & \multicolumn{10}{|c|}{ fraction } \\
\hline & 1 & 2 & 3 & 4 & 5 & 6 & 7 & 8 & 9 & 10 \\
\hline drawing ratio $\lambda_{\text {d.r. }}$ & 2.35 & 2.75 & 2.30 & 2.85 & 2.50 & 3.00 & 2.50 & 3.05 & 2.30 & 2.90 \\
\hline
\end{tabular}
long $\mathrm{T}_{2}$ component

$\begin{array}{llllllllll}0.24 & 0.15 & 0.27 & 0.09 & 0.15 & 0.09 & 0.38 & 0.07 & 0.27 & 0.07\end{array}$

* Sample 1 and 2 are from same lot number 1

Sample 3 and 4 are from same lot number 2 .

Sample 5 and 6 are from same lot number 3 .

Sample 7 and 8 are from same lot number 4

Sample 9 and 10 are from same lot number 5 .

Table IV. The fractions of the short $\mathrm{T}_{2}$ component and the long $\mathrm{T}_{2}$ component obtained from deconvolution of solid-echo ${ }^{1} \mathrm{H}$ FID signal as a function of drawing ratio at $160^{\circ} \mathrm{C}$

\begin{tabular}{lcccccccccc}
\hline & \multicolumn{10}{c}{ fraction } \\
sample number & 1 & 2 & 3 & 4 & 5 & 6 & 7 & 8 & 9 & 10 \\
drawing ratio $\lambda_{\text {d.r. }}$ & 2.35 & 2.75 & 2.30 & 2.85 & 2.50 & 3.00 & 2.50 & 3.05 & 2.30 & 2.90 \\
\hline short $\mathrm{T}_{2}$ component & & & & & & & & & & \\
& 0.52 & 0.55 & 0.55 & 0.64 & 0.56 & 0.58 & 0.51 & 0.59 & 0.53 & 0.57 \\
long $\mathrm{T}_{2}$ component & & & & & & & & & & \\
& 0.48 & 0.45 & 0.45 & 0.36 & 0.44 & 0.42 & 0.49 & 0.41 & 0.47 & 0.43
\end{tabular}

of 17-20 $\mu$ s. On the other hand, the ${ }^{1} \mathrm{H} \mathrm{T}_{2}$ values of the long $\mathrm{T}_{2}$ component are monotonously and largely increased in going to 120 to $200^{\circ} \mathrm{C}$ as compared with the short $\mathrm{T}_{2}$ component and the drawing ratio dependence of ${ }^{1} \mathrm{H} \mathrm{T}_{2}$ is very large. The ${ }^{1} \mathrm{H} \mathrm{T}_{2}$ values of the long $\mathrm{T}_{2}$ component become longer with an increase in the draw ratio. The slope of the plots of ${ }^{1} \mathrm{H} \mathrm{T}_{2}$ against temperature becomes very small with an increase in the drawing ration. These ${ }^{1} \mathrm{H} \mathrm{T}_{2}$ behavior may come from dynamic change through structural change by temperature variation as will be discussed.

Here, we must consider the amorphous structure that there exist two kinds of the mobile and immobile amorphous regions with different degree of free volume in which the PGA segments can undergo relatively fast and slow motions, respectively. This leads to the consideration that the short $\mathrm{T}_{2}$ component comes from the crystalline region and the immobile amorphous region, and the long $\mathrm{T}_{2}$ component comes from the mobile amorphous region. Thus, from the above experimental results, it is considered that the immobile amorphous region is changed to the mobile amorphous region in going from 120 to $160^{\circ} \mathrm{C}$. For this, the short $\mathrm{T}_{2}$ component at $160^{\circ} \mathrm{C}$ may be mainly contributed by the crystalline region. Therefore, it may be reasonable to consider that the fraction of the short $\mathrm{T}_{2}$ component at $160^{\circ} \mathrm{C}$ is very close to the crystallinity determined by WAXD. It can be said that large difference $(=0.35)$ between the crystallinity obtained by WAXD and the fraction of short $\mathrm{T}_{2}$ component obtained by ${ }^{1} \mathrm{H}$ solid-echo $\mathrm{NMR}$ at $120^{\circ} \mathrm{C}$ may come from the immobile amorphous region in which the segments in highly drawn PGA fiber do not 
contribute to the long $\mathrm{T}_{2}$ component even at $120^{\circ} \mathrm{C}$ because they are under strong restraint, that is, there does not exist enough large free volume to undergo fast motion. Thus, in highly drawn PGA fiber the significant amount of the immobile amorphous region exists and contributes to the short $T_{2}$ component in solid-echo ${ }^{1} \mathrm{H}$ FID signal. Further, it can be said that the crystalline peak in the observed WAXD patterns comes from the relatively long-range crystalline region, but the short $\mathrm{T}_{2}$ component in solid-echo ${ }^{1} \mathrm{H}$ FID signals comes from the relatively immobile amorphous region in addition to the crystalline region at $120^{\circ} \mathrm{C}$, and it is expected that the fraction of the mobile amorphous region decreases with an increase in the drawing ratio and then the short $\mathrm{T}_{2}$ component is increased.

Judging from the ${ }^{1} \mathrm{H} \mathrm{T}_{2}$ value for the long $\mathrm{T}_{2}$ component at $120^{\circ} \mathrm{C}$, it is expected that the segmental motion of PGA is much slower as compared with that of the amorphous region of polyethylene. ${ }^{18}$ Earlier pulse ${ }^{1} \mathrm{H}$ NMR study on molecular motion of polyethylene indicates that there are three kinds of regions such as the crystalline region, the non-crystalline(amorphous) region and the intermediate region linked between the crystalline region and the non-crystalline region. It is known that in the intermediate region the mobility is in between the non-crystalline region and the crystalline region. The immobile amorphous region of drawn PGA fiber as mentioned above may be corresponding to the intermediate structure like the polyethylene, of which the mobility is close to that in the crystalline region. PGA is different from polyethylene in chemical structure. Nevertheless, it is not unreasonable to accept a structural picture like polyethylene that PGA has the three kinds of regions. This consideration will be used to explain reasonably the NMR data of the PGA fibers as a function of temperature and the drawing ratio below.

Solid-echo ${ }^{1} \mathrm{H}$ FID signals were observed afresh for three kinds of as-spun PGA fiber and drawn PGA fibers with $\lambda_{\text {d.r. }}=$ 2.5 and 3.05, which were prepared from the same lot. The fraction of the short $\mathrm{T}_{2}$ component of as-spun PGA fiber as obtained from deconvoluted ${ }^{1} \mathrm{H}$ FID signal was plotted against temperatures in the temperature range from 80 to $200^{\circ} \mathrm{C}$, as

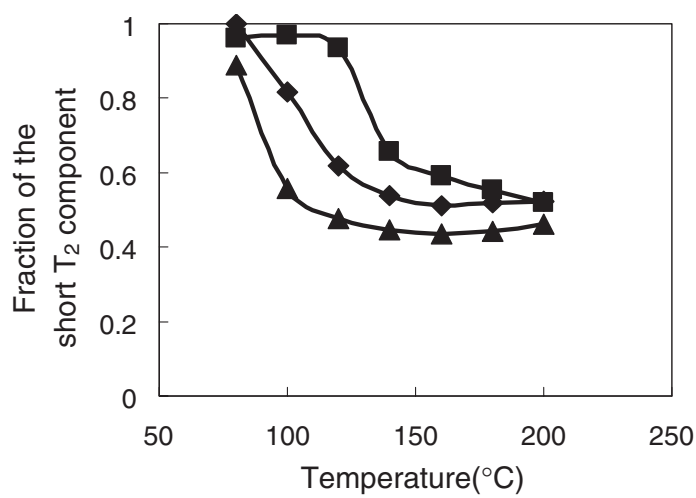

Figure 7. Plots of the fractions of the short $T_{2}$ component for as-spun PGA fiber $(\boldsymbol{\Delta})$, drawn PGA with the drawing ratio, $\lambda_{\text {d.r. }}=2.5(\bullet)$ and drawn PGA with the drawing ratio, $\lambda_{\mathrm{d} r \mathrm{r}}=3.05(\square)$ as estimated from deconvolution of the observed ${ }^{1} \mathrm{H}$ FID signals as a function of temperature in the temperature range from 80 to $200^{\circ} \mathrm{C}$. shown in Figure 7. As-spun PGA fiber is re-crystallized at $70{ }^{\circ} \mathrm{C}$ as observed by DSC. The fraction of the short $\mathrm{T}_{2}$ component is largely decreased with an increase in temperature in the temperature range from 80 to $120^{\circ} \mathrm{C}$. This shows that the PGA segments in the immobile amorphous region of recrystallized as-spun fiber become mobile in going to the mobile amorphous region by temperature elevation, and then the fraction of the short $\mathrm{T}_{2}$ component is largely decreased. In the temperature range from 120 to $200^{\circ} \mathrm{C}$, the fraction of the short $\mathrm{T}_{2}$ component becomes a plateau. The initial temperature of its plateau is defined by $\mathrm{T}_{\text {initial }}$. Therefore, it can be said that in this temperature range the short $\mathrm{T}_{2}$ component almost comes from the crystalline region. On the other hand, the fraction of the short $\mathrm{T}_{2}$ component for drawn PGA fiber with $\lambda_{\text {d.r. }}=2.5$ is decreased with an increase in temperature from 80 to $120^{\circ} \mathrm{C}$. In the temperature range from 160 to $200{ }^{\circ} \mathrm{C}$, the fraction of the short $\mathrm{T}_{2}$ component is almost independent of temperature. This behavior is very similar to that of as-spun PGA fiber except $T_{\text {initial }}$ shifts to higher temperature. Such a temperature shift in $\mathrm{T}_{\text {initial }}$ comes from the effect of drawing by which molecular motion of PGA segments in the immobile amorphous region is strongly restrained because of reduction of the free volume. In the case of drawn PGA fiber with $\lambda_{\text {d.r. }}=3.05$, the behavior is very different from that of as-spun PGA fiber and drawn PGA fiber with $\lambda_{\text {d.r. }}=2.5$. In the temperature range from 80 to $120^{\circ} \mathrm{C}$, the fraction of the short $\mathrm{T}_{2}$ component is almost independent of temperature. This shows that molecular motion of PGA segments in the immobile amorphous region is strongly restrained by high drawing, and then temperature of inducing a transitional change from the immobile amorphous region to the mobile amorphous region shifts to higher temperature side. Therefore, the fraction of the short $\mathrm{T}_{2}$ component is almost independent of temperature. At $120^{\circ} \mathrm{C}$ the immobile amorphous region suddenly begins to melt, and above $120^{\circ} \mathrm{C}$ changes transitionally to mobile state. Therefore, it can be said that $\mathrm{T}_{\text {initial }}$ depends on the degree of mobility of PGA segments in the immobile amorphous region.

From the above-mentioned experimental results, we draw a schematic diagram of the structure for as-spun PGA fiber(recrystallized and undrawn fiber) and drawn PGA fiber as shown in Figure 8. It can be said that in as-spun PGA fiber there does not exist almost the immobile amorphous region, and there exist the mobile amorphous region (A region) and crystalline region ( $\mathrm{B}$ region), and that in drawn PGA fibers there are the mobile amorphous region (A region) as the minor component, the immobile amorphous region ( $\mathrm{C}$ region), and crystalline region (B region) as the major component.

\section{Hydrolysis Mechanism of PGA Fibers}

The hydrolysis mechanism of PGA fibers has been elucidated on the basis of solid-echo ${ }^{1} \mathrm{H}$ NMR results. It is known that the hydrolysis of PGA initiates from the amorphous region. ${ }^{14,21,22}$ It is thought that water molecules penetrate into the amorphous region and occupy in the available free volume, and then hydrolysis reaction occurs. It can be said that the fraction of the free volume occupied by water molecules in 


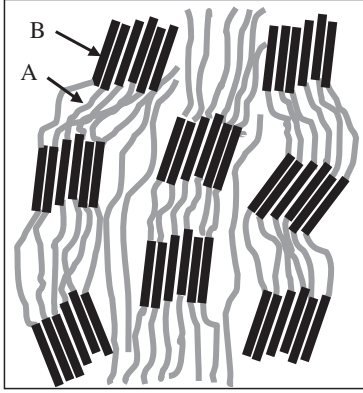

as-spun fiber

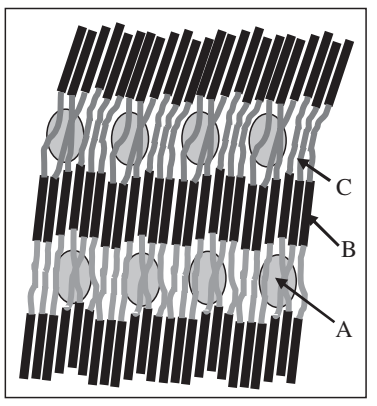

drawn fiber
Figure 8. A possible schematic diagrams for the structure of as-spun and drawn PGA fibers. A: mobile amorphous region, B: crystalline region and $\mathrm{C}$ : immobile amorphous region.

PGA fibers is decreased with an increase in the drawing ratio. Then, it is suggested that the hydrolysis rate of PGA depends on the amount of the free volume, that is, the fraction of the amorphous region. For this, it is very important to estimate exactly the fraction. As above-mentioned, we have characterized structure and dynamics of the amorphous component consisting of the mobile amorphous region and the immobile amorphous region of PGA fibers by means of pulse ${ }^{1} \mathrm{H}$ NMR. It has been clarified that PGA segments in the mobile amorphous region are undergoing very fast motion, and in the immobile amorphous region are undergoing relatively slow motion in strong restrained state, and at higher temperatures the immobile amorphous region is changed to the mobile amorphous region as above-mentioned.

In order to elucidate the hydrolysis rate of PGA fibers, the significant quantity such as the fraction of the immobile amorphous region associated with the hydrolysis rate has been adopted. In as-spun PGA fiber, the fraction of the short $\mathrm{T}_{2}$ component is almost a constant at temperatures above $120^{\circ} \mathrm{C}$. On the other hand, in drawn PGA fibers the fraction of the short $\mathrm{T}_{2}$ component approaches to almost a constant at $160^{\circ} \mathrm{C}$. The difference between the fractions of the short $\mathrm{T}_{2}$ components at 120 and $160{ }^{\circ} \mathrm{C}$ may be approximately equal to the fraction of the immobile amorphous component ( $\mathrm{C}$ region). Thus, it can be said that even if the crystallinities of drawn PGA fibers are almost equal to each other, the fractions of the immobile amorphous region ( $\mathrm{C}$ region) in corresponding fibers are different from each other. This has been clarified from the experimental results by ${ }^{1} \mathrm{H}$ solid-echo NMR. It is expected from the experimental finding that the tensile strength of drawn PGA fibers after hydrolytic acceleration test depends not only on the fraction of the crystalline region, but also on the fraction of the immobile amorphous region. According to such a consideration we will clarify the relationship between the tensile strength after hydrolytic acceleration test and the fraction of the immobile amorphous region as will be described below.

Figure 9 shows the relationship between the ratio of the tensile strength after hydrolytic acceleration test to that before hydrolytic acceleration and the fraction of the immobile

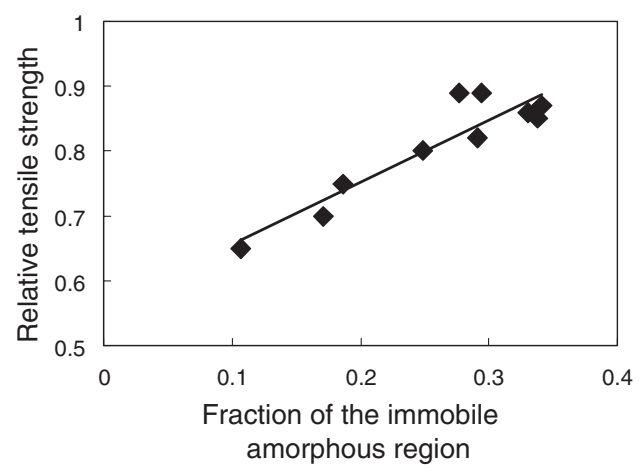

Figure 9. The relationship between the ratio of the tensile strength after hydrolytic acceleration test to that before hydrolytic acceleration for 10 kinds of drawn PGA fibers and the fraction of the immobile amorphous region.

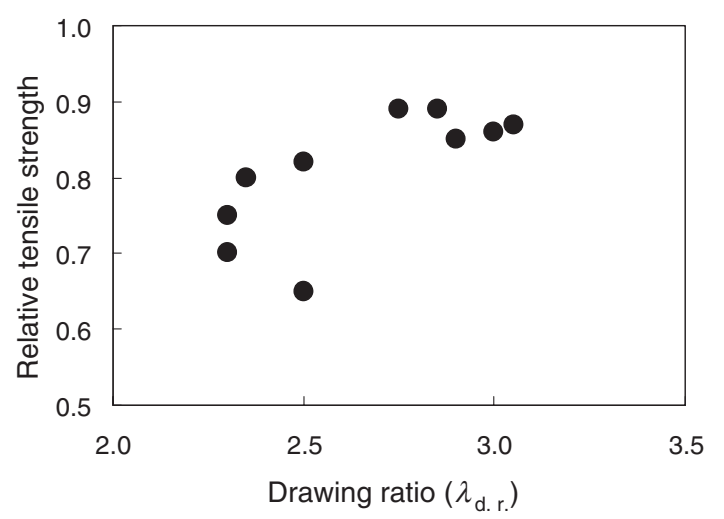

Figure 10. The relationship between the ratio of the tensile strength after hydrolytic acceleration test to that before hydrolytic acceleration for 10 kinds of drawn PGA fibers and the drawing ratio.

amorphous region as obtained from 10 kinds of drawn PGA fibers with different drawing ratios, which were obtained from the difference between the fractions of the short $\mathrm{T}_{2}$ components at 120 and $160{ }^{\circ} \mathrm{C}$. It is seen from this figure that the experimental data lie on a straight line. This means that there is the close relationship between the tensile strength and the fraction of the immobile amorphous region, and then if the fraction of the immobile amorphous region for drawn PGA fiber is obtained by solid-echo ${ }^{1} \mathrm{H}$ NMR, the tensile strength of drawn PGA fibers can be exactly determined. Figure 10 shows the relationship between the ratio of the tensile strength after hydrolytic acceleration test to that before hydrolytic acceleration for PGA fibers and the drawing ratio. The two experimental data of the tensile strength for two kinds of drawn PGA fibers with $\lambda_{\text {d.r. }}=2.5$ are plotted against the fraction of the immobile amorphous region as obtained by above-mentioned estimation. These two experimental data are different from each other. Two kinds of drawn PGA fibers were prepared from different lots and thus the ratio of the tensile strength after hydrolytic acceleration test to that before hydrolytic acceleration for them were 0.82 and 0.65 . Probably, this may come from different molecular weight and its distribution of original PGA, and different conditions such as 
drawing temperature and drawing speed of two kinds of PGA fibers used in lots. Therefore, this leads to the different ratio of the tensile strength after hydrolytic acceleration test to that before hydrolytic acceleration for PGA fibers, even if the drawing ratios for drawn PGA fibers are the same. However, the fractions of the immobile amorphous regions were different from each other. From the above experimental results, it can be said that the hydrolysis rate of fibers is a very important factor for the bioabsorbable sutures.

\section{CONCLUSIONS}

Higher-order structure of as-spun and highly-drawn PGA fibers has been reasonably characterized by solid-echo ${ }^{1} \mathrm{H}$ NMR and WAXD methods. From analysis of the observed WAXD pattern of highly-drawn PGA fibers, the crystallinities of highly-drawn fibers with $\lambda_{\text {d.r. }}$ of $2.5,2.8$ and 3.2 are determined to be $0.50,0.51$ and 0.52 , respectively. This shows that the crystallinities of the PGA fibers with different drawing ratio are very close to each other. On the other hand, the observed solid-echo ${ }^{1} \mathrm{H}$ NMR FID signals of as-spun and drawn PGA fibers show a multi-component decay consisting of the short $\mathrm{T}_{2}$ component and the long $\mathrm{T}_{2}$ component. From the experimental results, the short $\mathrm{T}_{2}$ component was assigned to the crystalline region and the immobile amorphous region, and the long $\mathrm{T}_{2}$ component was assigned to the mobile amorphous region. It was found that the fractions of the short $T_{2}$ component and the long $\mathrm{T}_{2}$ component of highly-drawn fibers largely depend on the drawing ratio $\lambda_{\text {d.r. }}$ and temperature, and that the fractions of the short $\mathrm{T}_{2}$ component for drawn PGA fibers with $\lambda_{\text {d.r. }}$ of 2.5 at $120^{\circ} \mathrm{C}$ and $160^{\circ} \mathrm{C}$ are 0.85 and 0.56 , respectively. Further, it was found that the fractions of the short $\mathrm{T}_{2}$ component for the drawn fibers above $160{ }^{\circ} \mathrm{C}$ are very close to the crystallinities determined by the WAXD patterns, but the fractions of the short $\mathrm{T}_{2}$ component at $120^{\circ} \mathrm{C}$ are different from each other. The structural characterization was used to understand mechanical property of the drawn PGA fibers. Then, it was found that the fraction of the corresponding amorphous region is closely related to the ratio of the tensile strength after hydrolytic acceleration test to that before hydrolytic acceleration for PGA fibers.

Acknowledgment. The authors are grateful to H. Kanno and M. Koyama of Mitsui Chemical Analysis \& Consulting
Service, Inc. for the WAXD and ${ }^{1} \mathrm{H}$ NMR analyses, and also are grateful to Professor Emeritus H. Saito of Himeji Institute of Technology for his useful comments on the manuscript. T. Asakura acknowledges financial support from a Grant-inAid for Scientific Research from the Ministry of Education, Science, Sports and Culture of Japan (18105007).

Received: December 13, 2006 Accepted: September 18, 2007 Published: November 20, 2007

\section{REFERENCES}

1. D. K. Gilding and A. M. Reed, Polymer, 20, 1459 (1979).

2. J. Blomqvist, B. Mannfors, and L. O. Pietila, Polymer, 43, 4571 (2002).

3. B. X. Fu, B. S. Hsiao, G. Chen, J. Zhou, I. Koyfman, D. D. Jamiolkowski, and E. Dormier, Polymer, 43, 5527 (2002).

4. C. Nakafuku and H. Yoshimura, Polymer, 45, 3583 (2004).

5. H. Nishida, M. Yamashita, T. Endo, and Y. Tokiwa, Macromolecules, 33, 6982 (2000).

6. K. K. Yang, X. L. Wang, and Y. Z. Wang, J. Macromol. Sci., Part C-Polym: Rev., C42, 373 (2002).

7. S. A. Sabino, S. Gonzalez, L. Marquez, and L. Feijoo, Polym. Degrad. Stab., 69, 209 (2000).

8. C. P. Ooi and R. E. Cameron, J. Biomed. Mater. Res., Appl. Biomat., 63, 280 (2002).

9. J. R. Sarasua, R. E. Prud'homme, M. Wisniewski, A. L. Borgne, and N. Spassky, Macromolecules, 31, 3895 (1998).

10. J. F. Mano, J. L. Gomez Ribelles, N. M. Alves, and M. S. Sanchez, Polymer, 46, 8258 (2005).

11. H. Hu and D. L. Dorset, Macromolecules, 23, 4604 (1990).

12. D. K. Gilding and A. M. Reed, Polymer, 20, 1459 (1979).

13. B. X. Fu, B. S. Hsiao, G. Chen, J. Zhou, I. Koyfman, D. D. Jamiolkowski, and E. Dormier, Polymer, 43, 5527 (2002).

14. C. C. Chu, J. Appl. Polym. Sci., 26, 1727 (1981).

15. Y. Chatani, K. Suehiro, Y. Okita, H. Tadokoro, and K. Chujo, Makromol. Chem., 113, 215 (1968).

16. H. Montes de Oca, I. M. Ward, P. G. Klein, M. E. Ries, J. Rose, and D. Farrar, Polymer, 45, 7261 (2004).

17. "Solid State NMR of Polymers," I. Ando and T. Asakura, Ed., Elsevier Science, Amsterdam, 1998.

18. H. Uehara, T. Yamanobe, and T. Komoto, Macromolecules, 33, 4861 (2000).

19. C. Marega, A. Marigo, R. Zannetti, and G. Paganetto, Eur. Polym. J., 28, 1485 (1992).

20. Z. G. Wang, B. S. Hsiao, F. Yeh, J. Zhou, E. Dormier, and D. D. Jamiolkowski, Polymer, 41, 621 (2000).

21. S. Hurrell and R. E. Cameron, Biomaterials, 23, 2401 (2002).

22. C. C. Chu and M. Louie, J. Appl. Polym. Sci., 30, 3133 (1985). 